\title{
Introduction to special celiac section
}

\author{
Jeff L. Fidler \\ Mayo Clinic, Rochester, MN, USA
}

I am honored to be invited to serve as a Guest Editor for a special section on the imaging of celiac disease in this month's journal of Abdominal Radiology.

Celiac disease is an immune-mediated inflammatory enteropathy triggered by gluten exposure in genetically susceptible individuals. It is estimated that celiac disease affects approximately $1 \%$ of the US population, and the incidence has steadily risen over the last several years. Therefore, most abdominal radiologists will encounter this disease in their clinical practice.

Clinicians are becoming more aware of this disease and when patients present with the classic signs and symptoms of malabsorption referral to gastroenterology, and subsequent diagnosis can be made with serological testing and endoscopic biopsy. However, a large number of patients may exhibit atypical symptoms which can lead to a delay in diagnosis and development of multisystemic complications.

Cross-sectional imaging including $\mathrm{CT}$ and $\mathrm{MR}$ and dedicated small bowel imaging with enterography technique is being more widely utilized in patients with abdominal symptoms. Awareness of the findings of celiac disease and the associated complications may allow the radiologist to be the first to suggest the diagnosis. In patients with known celiac disease, cross-sectional imaging is also used for the evaluation of the development of complications occurring in patients with refractory celiac disease. Therefore, it is imperative that radiologists be familiar with these complications and their appearances.
In this edition, there are several articles which will improve the radiologist's understanding of this unique disease. In the first article, gastroenterologists who are international experts in celiac disease provide a state-ofthe-art update and review of the important clinical issues including background, demographics, diagnosis, treatment, and complications. Sheedy et al. provide an extensive pictorial review of the imaging findings and complications that occur in celiac disease. Finally, two scientific studies assess the imaging findings in refractory celiac disease and collagenous sprue which may mimic conventional celiac disease. I hope the readers find these articles to be educative and helpful in improving their knowledge regarding celiac disease.

Compliance with ethical standards

Funding No funding was received for this study.

Conflict of interest The author declares that he has no conflict of interest.

Ethical approval This article does not contain any studies with human participants performed by any of the authors.

Informed consent Statement of informed consent was not applicable since the manuscript does not contain any patient data.

Correspondence to: Jeff L. Fidler; email: fidler.jeff@mayo.edu 